\title{
HISTOLOGICAL FEATURES OF THE ATRIAL MYOCARDIUM AND SCALLOP MUSCLES OF ANGLO-NUBIAN GOAT (Capra aegagrus hircus)
}

\author{
Victor KHVATOV and Mikhail SHCHIPAKIN ${ }^{\star 凶}$ \\ Saint Petersburg State University of Veterinary Medicine, 5, Chernigovskaya st., St. Petersburg 196084, Russia \\ Email: shipakin@spsuvm.ru; (D)RCiD: 0000-0002-2960-3222 \\ Supporting Information
}

\begin{abstract}
This study presents results about features of the structure of the atrial myocardium and scallop muscles of the Anglo-Nubian goat (Capra aegagrus hircus), and their morphometric parameters have been determined. Sixteen corpses of Anglo-Nubian goats at the age of twelve months were studied in the Department of Animal Anatomy of the St. Petersburg State University of Veterinary Medicine. The study was carried out by making histological preparations. The atrial myocardium of Anglo-Nubian goats consists of working cardiomyocytes, tightly adjacent to each other, between which capillary blood vessels and single adipocytes are revealed. It has been determined that atrial cardiomyocytes in Anglo-Nubian goats are characterized by heterogeneity of their structure, and secretory granules and perinuclear clearances are found in cardiomyocytes less differentiated in terms of contractile activity. In the present study, the histological structure of the atrial scallop muscles of the Anglo-Nubian goat was identified. Also, the atrial myocardium's working cardiomyocytes' morphometric parameters and its scallop muscles had discovered. The thickness of the endocardium in these sections was measured by determining the diameters of its nuclei. The obtained data indicate that the histological structure of the atrial myocardium and its scallop muscles in Anglo-Nubian goats has possibly similar features in compared with small ruminants from commercial breeds (sheep and goat). These results expand the field of knowledge on pedigree anatomy and histology of the heart in farm animals, particularly in small ruminants.
\end{abstract}

Keywords: Atrium, Goat, Histology, Myocardium, Scallop muscles.

\section{INTRODUCTION}

The myocardium is the heart's main shell, from the functional state of which its contractile ability consists (DiVincenti et al., 2014; Emam and Abugherin, 2019). Knowledge of its macro-anatomy and histological structure is essential and imperative for any practicing veterinarian. Almost any heart disease is accompanied in one way or another by a change in the biochemical structure of the myocardium, thereby causing its pathology (Frandson et al., 2009).

Studies aimed at evaluating the histostructure of the heart myocardium in animals in terms of species and also species expand the area of knowledge of veterinary specialists, which allows the most accurate and individual surgical and therapeutic treatment of animals with cardiac pathologies, which is more important issue in farm animal veterinary (Buczinski et al., 2010).

Anglo-Nubian goats (Capra aegagrus hircus) are distinguished by their high body weight characteristics, have an excellent milk yield level, and are also valued for the quality of dairy and meat products in Russian Federation (Kuga, 2012). The atrial myocardium in farm animals includes two layers - superficial and deep, connected by tight muscle anastomoses (Kuga, 2012). According to König and Libich (2004), the atrial myocardium's superficial layer in small and in cattle it is circular and common to both the right and left atria, and the circular layer is located from one ear to the other. According to Kozlovskaya, (2014), Ateş et al. (2017), and Emam and Abugherin (2019), the second layer of the atrial myocardium is deep, and its muscle fibers have an oblique-longitudinal direction. This layer is individual for each atrium and starts from the fibrous rings in the atrioventricular opening area and ends in the superficial layer's muscle fibers.

The atria in ruminants from the inside have the appearance of a sponge due to the scallop muscles, which contribute to the complete squeezing of blood during the chamber's contraction. The scallop muscles are absent in the interatrial septum (Maximilian Buja, 2007). According to Huxley and Haselgrove (1977) his number of scallop muscles on the inner surface of the heart of animals and humans is relatively constant, and the intervals between them decrease in the direction from the base of the ear to its apex.

The scallop muscles in domestic animals are divided into muscles of the first and second order, the first of which has a large diameter and are located perpendicularly and slightly obliquely to the ear's longitudinal axis (Hooper and Thuma, 2005). The second ones depart at an angle from the first order's muscles and are significantly inferior in diameter. In cattle, the scallop muscles are of moderate severity (Vansyatskaya and Kirpaneva, 2014).

In the studied literature, there was not any completive and strength information on the atrial myocardium's histological structure and their scallop muscles in small ruminants, namely, in Anglo-Nubian goats. Thus, this study's 
purpose was to investigate and evaluate the histological structure peculiarities of the atrial myocardium and their scallop muscles of an Anglo-Nubian goat and establish the morphometric data of the histological structures of the scallop muscles and cardiac muscle of the atria.

\section{MATERIALS AND METHODS}

There were used dated materials for the study obtaining during slaughter from the farm "Gzhel Podvorie" in the Russian Federation's Moscow region. The dated material was presented by sixteen corpses of Anglo-Nubian goats older than one year.

The material was fixed in a 10\% solution of neutral formalin for 24 hours (Gushchin and Muzhikyan, 2014), after which it was embedded in paraffin according to the generally accepted technique (Muzhikyan et al., 2014). Then sections with a thickness of 3-5 $\mu \mathrm{m}$ were made, stained with hematoxylin and eosin. According to McManus, some of the sections were stained with Trichrome according to Masson, toluidine blue, and Schiff-iodic acid. The histological preparations analysis was carried out using a Carl Zeiss Axioskop 2 Plus light-optical microscope (Germany) at a magnification of 40, 100, 400, 1000. Microphotography was performed using a digital camera (CarlZeiss Axio Cam ERc5s, Germany) and the AxioVision 4.8 software. Morphometric measurements were performed manually using the AxioVision 4.8 software (Image J, Germany).

\section{Statistical analysis}

The variational-statistical processing of the research results was carried out on IBMPC / AT and "Pentium IV" in Windows 2000, using the data analysis package in the program "Excel Windows Office XP" and "Statistika 6.0" (Statsoft, USA) with the calculation of the arithmetic mean and its standard error $(M \pm m)$. In the statistical analysis of the data obtained, the Student's t-test was used for independent samples, while the differences were considered significant at $p<0.05$. All anatomical and histological terms correspond to the "International Veterinary Anatomical Nomenclature," fifth edition, translation and Russian terminology of Zelenevsky (2013) and International histological nomenclature edited by Semchenko et al. (1999).

\section{Ethical regulation of study}

This research was conducted in accordance with tin accordance with ethical standards for the use of animals for scientific purposes, referred to Federal Law of 24.04.1995 N 52-FL (as amended on 08.12.2020) "On the animal world". The main research technique was chosen to study the microstructure of the atrial myocardium and scallop muscles - the production of histological preparations for sampling the material under study on fresh hearts of Anglo-Nubian goats. The material was taken by the method of fine anatomical preparation (Minutes No. 134 dated 09/15/2020).

\section{RESULTS AND DISCUSSION}

The atrial myocardium of an Anglo-Nubian goat is formed by tightly adjacent contractile (working) cardiomyocytes, forming long multidirectional strands (Figures 1 and 2). A more tortuous course of muscle fibers and wider gaps between them are noted in the studied animals than in the interventricular septum. The contractile cardiomyocytes in this section are elongated. The cell thickness varies in the range of 16-20 microns and averages $17.6 \pm 1.5$ microns. The area of cells on a cross-section averages $294.5 \pm 31.6 \mu \mathrm{m}^{2}$. In general, in this part of the heart, cardiomyocytes are characterized by structural heterogeneity; in cells less differentiated in terms of contractile activity, perinuclear clearances and secretory granules are determined, which are not found in cardiomyocytes of the interventricular septum (Figure 3). The nuclei of cells are oval, slightly elongated, normochromic, with a uniform distribution of small-lumpy chromatin. In some of the cells, the nuclei are elongated, flattened, moderately hyperchromic. The average values for a large core diameter are 10.1 $\pm 1.1 \mu \mathrm{m}$, for a small one, $4.3 \pm 0.4 \mu \mathrm{m}$. The average area of the nuclei of contractile cardiomyocytes varies within $40-48$ $\mu \mathrm{m}^{2}$ and averages $42.7 \pm 3.2 \mu \mathrm{m}^{2}$.

According to Masson, staining with trichrome between the muscle elements of the myocardium, layers of loose, small-cell, unformed connective tissue of different thickness, containing capillary-type blood vessels and single small groups of adipocytes, are revealed (Figures 4 and 5). Along the periphery, thin cords of collagen fibers pass into a loose, unformed connective tissue of the endocardium, covered with one layer of flattened endothelial cells (Figure 6). The endocardium thickness in this section varies within 13-25 microns and averages $17.2 \pm 2.2$ microns. The nuclei of endothelial cells are predominantly oval and elongated. The large and small diameter of the endothelial cell nucleus averages $7.8 \pm 1.5$ and $4.8 \pm 0.6$ microns.

The scallop muscle in the Anglo-Nubian goat is formed by tightly adjacent contractile (working) cardiomyocytes, forming long multidirectional cords (Figures 7 and 8). The contractile cardiomyocytes in this section are elongated. The cell thickness varies within 12-24 microns and averages $18.9 \pm 1.6$ microns. The area of cells on a cross-section averages $278.3 \pm 24.1 \mu^{2}$. The nuclei of cells are oval, slightly elongated, normochromic, with a uniform distribution of smalllumpy chromatin. In some of the cells, the nuclei are elongated, flattened, moderately hyperchromic. The average values for the large diameter of the nucleus are $12.7 \pm 1.3 \mu \mathrm{m}$, for the small diameter $-4.2 \pm 0.4 \mu \mathrm{m}$. The average area of the nuclei of contractile cardiomyocytes varies within $40-45 \mu \mathrm{m}^{2}$ and averages $42.9 \pm 3.2 \mu \mathrm{m}^{2}$. 
Table 1 - Morphometric data of the histological structures of the atrial myocardium and their scallop muscles in Anglo-

Nubian goats, $\mu \mathrm{m}$.

Heart histostructure / Localization

Thickness of working cardiomyocytes

Average area of working cardiomyocytes, $\mu \mathrm{m}^{2}$

Average area of nuclei of working cardiomyocytes, $\mu \mathrm{m}^{2}$

Large diameter of the nuclei of working cardiomyocytes

Small diameter of the nuclei of working cardiomyocytes

\begin{tabular}{cc} 
Atrial myocardium & Atrial comb muscles \\
\hline $17.6 \pm 1.5$ & $18.9 \pm 1.6$ \\
$294.5 \pm 31.6$ & $278.3 \pm 24.1$ \\
$42.7 \pm 3.2$ & $42.9 \pm 3.2$ \\
$10.1 \pm 1.1$ & $12.7 \pm 1.3$ \\
$4.3 \pm 0.4$ & $4.2 \pm 0.4$
\end{tabular}

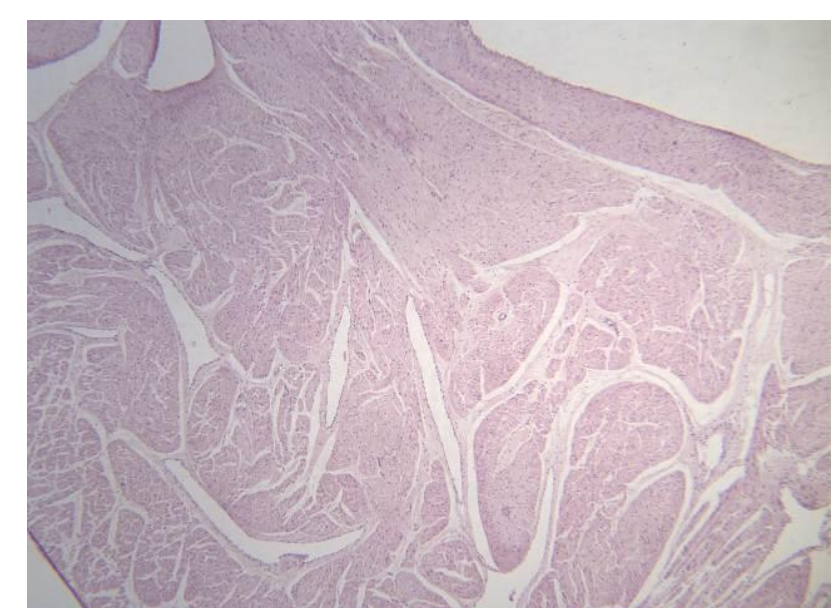

Figure 1 - Atrial myocardium. Hematoxylin-eosin staining. Magnification 40.

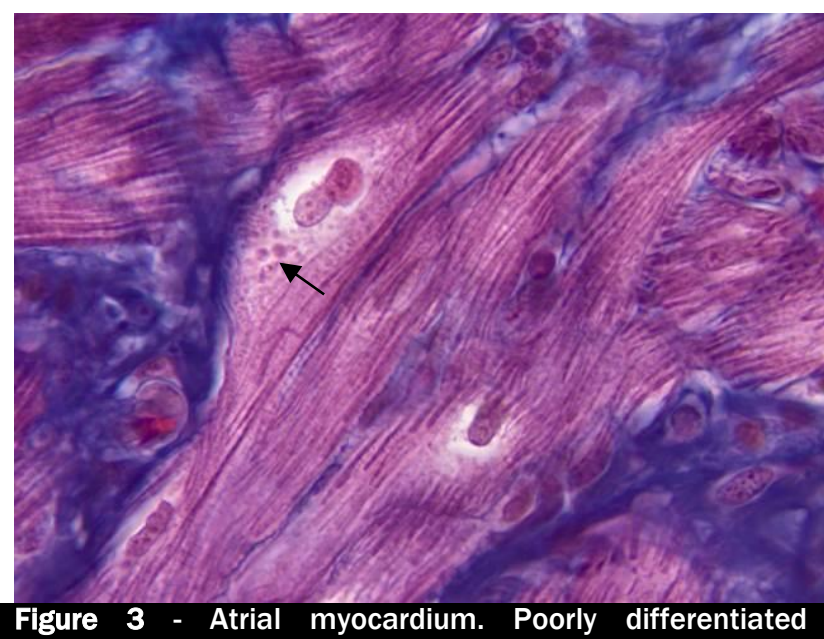

binucleated cardiomyocytes containing characteristic secretory granules (arrow) are revealed. Masson's trichrome staining. Magnification 400.

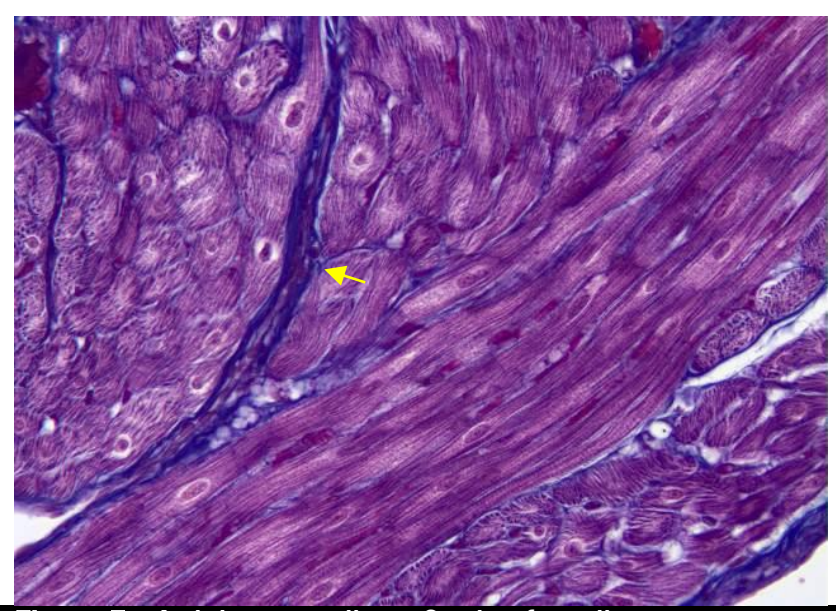

Figure 5 - Atrial myocardium. Cords of cardiomyocytes are separated by connective tissue layers stained with a blue dye (arrow). Masson's trichrome staining. Magnification 400.

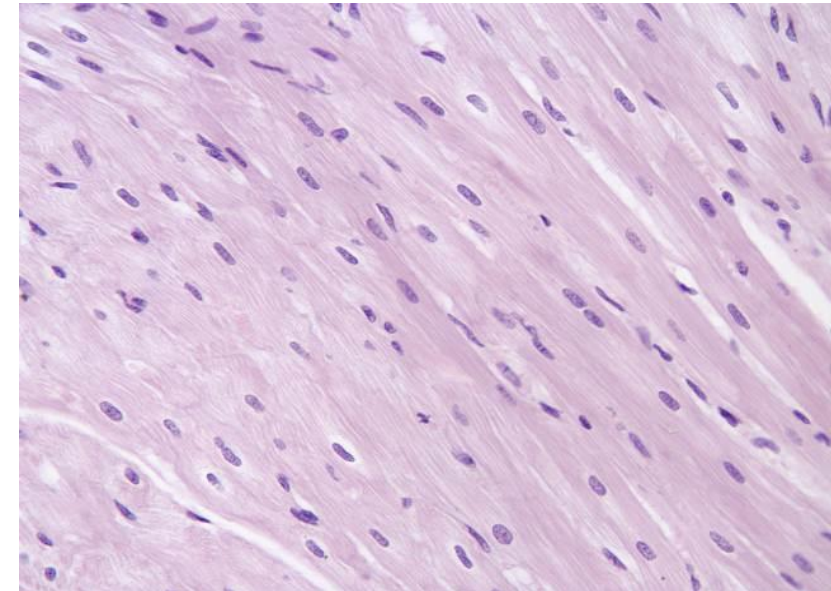

Figure 2 - Atrial myocardium. Cords of contractile (working) cardiomyocytes of typical structure are determined. Hematoxylin-eosin staining. Magnification 400.

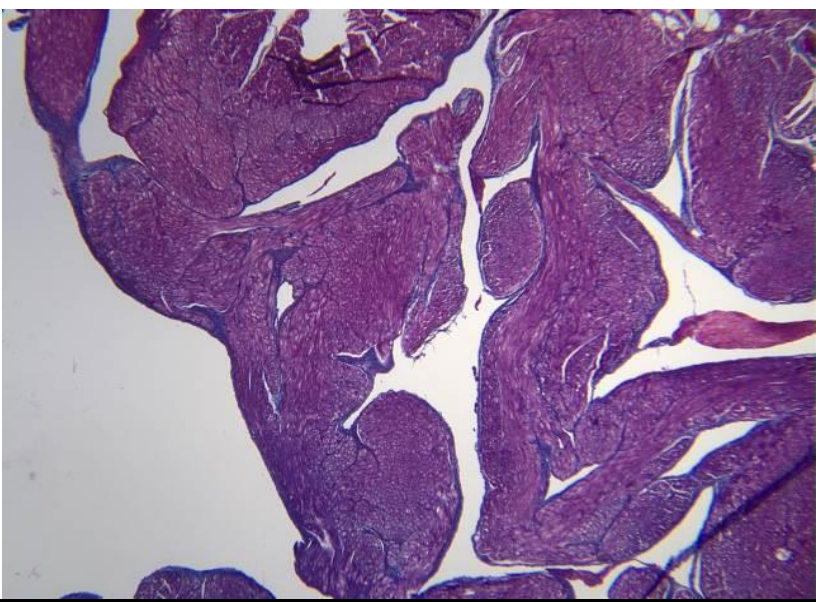

Figure 4 - Atrial myocardium. Masson's trichrome staining Magnification 40.

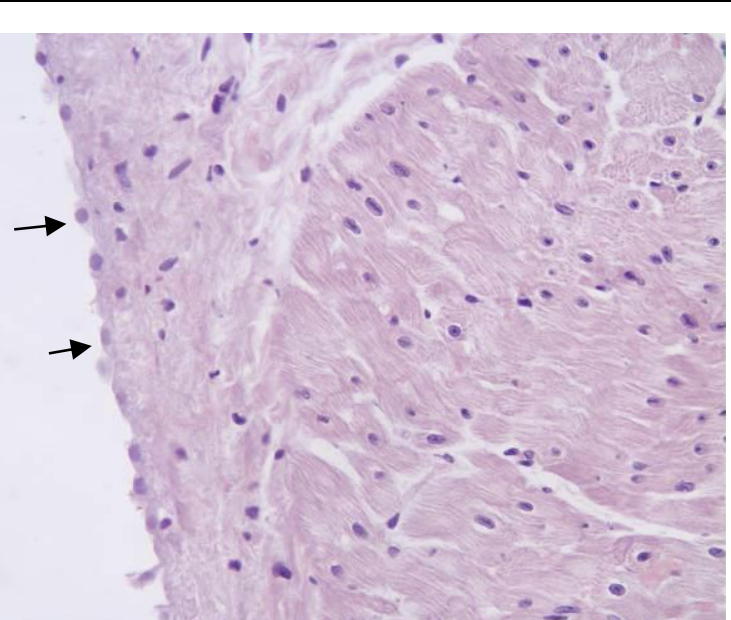

Figure 6 - Atrial myocardium. The strands of contractile (workers) are determined. The endocardium is lined with one layer of endothelial cells (arrows). Hematoxylin-eosin staining. Magnification 400. 
When staining with Masson's trichrome, between the muscle elements of the scallop muscle's myocardium, layers of loose, small-celled unformed connective tissue of varying thickness, containing capillary-type blood vessels and single small groups of adipocytes, are revealed (Figures 9 and 10). Along the periphery, thin cords of collagen fibers pass into a loose, unformed connective tissue of the endocardium, covered with one layer of flattened endothelial cells. The endocardium thickness in this section varies within 15-30 microns and averages $21.6 \pm 3.5$ microns. The nuclei of endothelial cells are predominantly oval and elongated. The large and small diameter of the endothelial cell nucleus averages $7.3 \pm 0.5 \mu \mathrm{m}$ and $3.1 \pm 0.3 \mu \mathrm{m}$ (Figures 11 and 12).

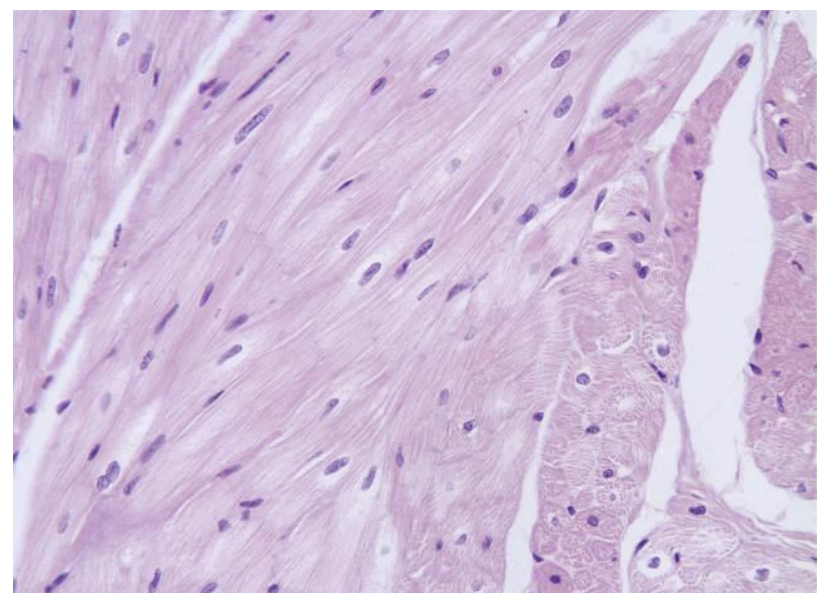

Figure 7 - The scallop muscle. The multidirectional strands of contractile (working) cardiomyocytes are determined. Hematoxylin-eosin staining. Magnification 400.

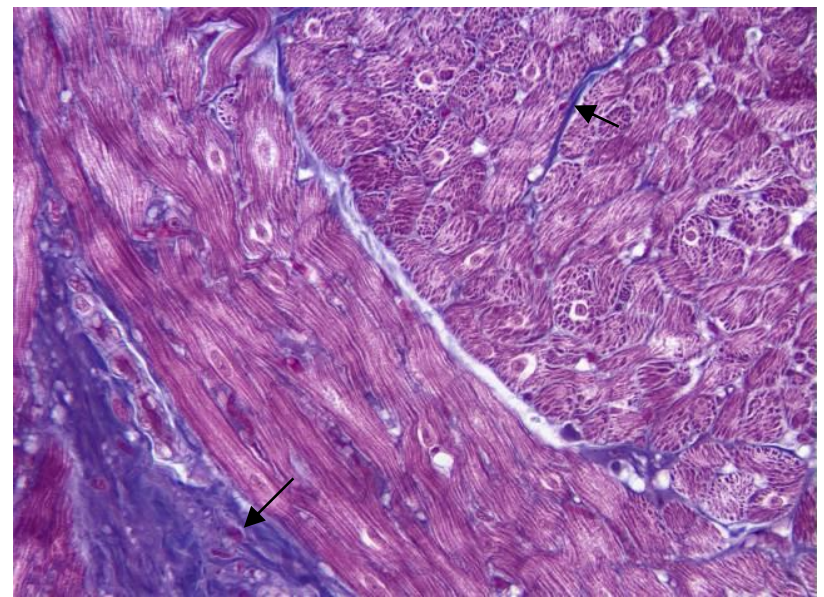

Figure 9 - The scallop muscle. Cords of cardiomyocytes are separated by connective tissue layers stained with a blue dye (arrow). Masson's trichrome staining. Magnification 400.

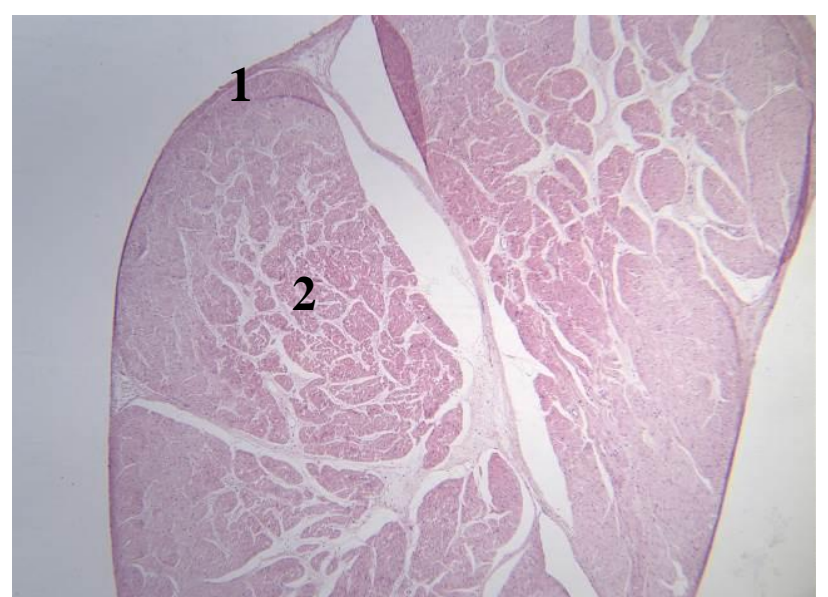

Figure 11 - The scallop muscle. Designations: endocardium (1), myocardium (2). Hematoxylin-eosin staining. Magnification 100.

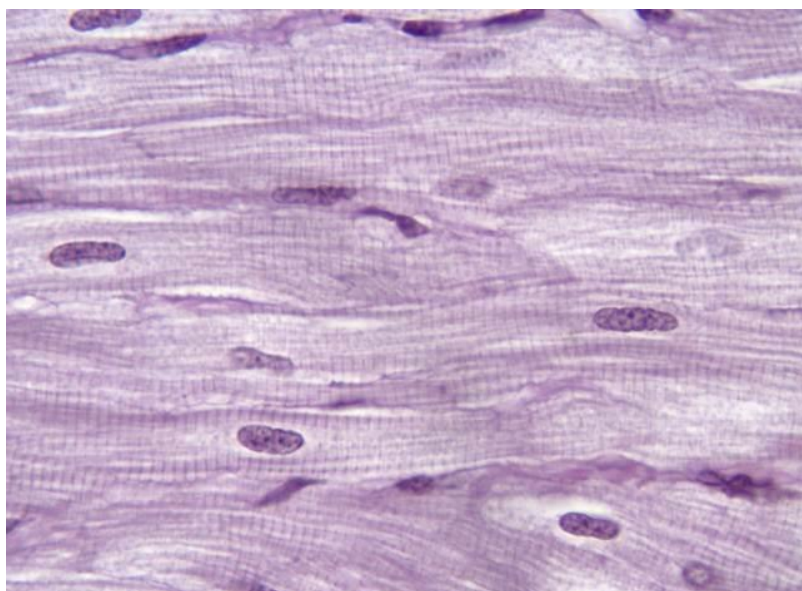

Figure 8 - The scallop muscle. Multidirectional strands of contractile (working) cardiomyocytes with a clearly expressed transverse striation are determined. Staining with Schiff-iodic acid according to McManus. Magnification 1000

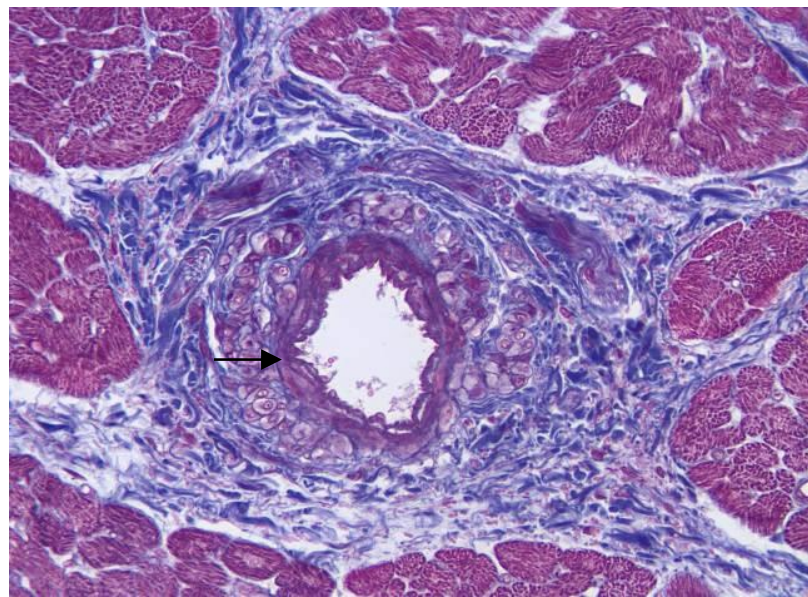

Figure 10 - The scallop muscle. In the thickness of the myocardium, a blood vessel (artery) of the muscle type (arrow) is determined, surrounded by loose connective tissue. Masson's trichrome staining. Magnification 400.

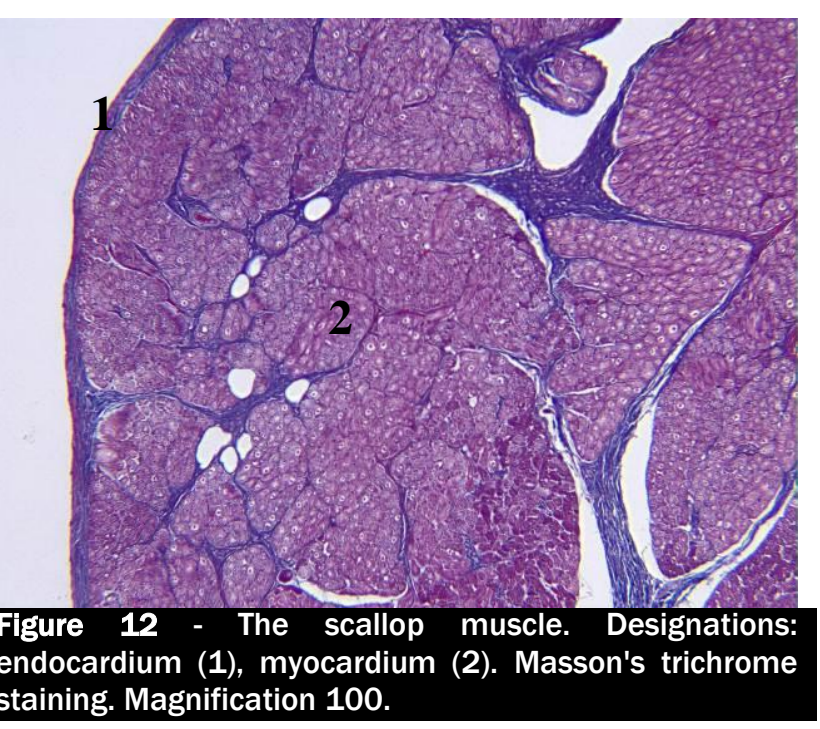


In some of related studies (Crick et al., 1980; Frandson et al., 2009; Emam and Abugherin, 2019), there was no contradict the data on the histology of the atrial myocardium in the report, and this study confirms these results. Findings of present study was in agreement with Endo et al. (1995) and Calkins et al. (2008) which identified the anatomy and histology of the atria of domestic and wild animals' hearts, and had same results on the scallop muscles' histology.

\section{CONCLUSION}

It was found that the atrial myocardium of Anglo-Nubian goats consists of working cardiomyocytes, tightly adjacent to each other, between which capillary-type blood vessels and single adipocytes are detected. As well as, atrial cardiomyocytes in Anglo-Nubian goats are characterized by heterogeneity of their structure. In less differentiated, in terms of contractile activity, cardiomyocytes, secretory granules, and perinuclear clearances are identified and reported. The histological structure of the scallop muscles was identified. It was determined that these heart structures consist of working cardiomyocytes, which in their structure are similar to the atrial myocardium. The morphometric parameters of the working cardiomyocytes of the atrial myocardium and its scallop muscles were identified in Anglo-Nubian goats. The data obtained indicate that possibly the histological structure of the atrial myocardium and its scallop muscles of AngloNubian goats has similar features inherent in goat or sheep species (there in no breed-specific variations). These results expand the field of knowledge on pedigree anatomy and histology of the heart in farm animals, particularly in small ruminants. The established morphometric parameters of the atrial myocardium and scallop muscles can be used as a theoretical basis by veterinary histologists, embryologists, and pathologists in the course of diagnosing heart pathologies in Anglo-Nubian goats.

\section{DECLARATIONS}

\section{Corresponding Author}

Email: shipakin@spsuvm.ru

\section{Authors' Contribution}

V.Kh. conceived of the presented idea and developed the theory and performed the computations. M.Sh. verified the analytical methods to investigate and supervised the findings of this work. All authors discussed the results and contributed to the final manuscript.

\section{Conflict of interests}

The authors have not declared any conflict of interests.

\section{Acknowledgements}

The study was carried out with the financial support of the Russian Foundation for Basic Research within the framework of scientific project No. 19-316-90033.

\section{REFERENCES}

Ateş S, Karakurum E, Takci L, Başak F, and Kúrtúl I (2017). Morphology of the atrioventricular valves and related intraventricular structures in the wild pig. Folia Morphology, 76(4): 650-659. DOI: https://doi.org/10.5603/fm.a2017.0051

Buczinski S, Rezakhani A, and Boerboom D (2010). Heart disease in cattle: diagnosis, therapeutic approaches and prognosis. Veterinary journal, 184(3): 258-263. DOI: https://doi.org/10.1016/j.tvjl.2009.05.005

Calkins H, Ho SY, Cabrera JA, Bella PD, Farre J, Kautzner J, and Tchou P (2008). Anatomy of the left atrium and pulmonary veins. Atrial Fibrillation Ablation: The State of the Art Based on the Venicechart International Consensus Document. Blackwell Publishing Ltd, UK. pp. 1-10. Doi: https://doi.org/10.1002/9780470692646.ch1

Crick SJ, Sheppard MN, Ho SY, Gebstein L, and Anderson RH (1998). Anatomy of the pig heart: comparisons with normal human cardiac structure. Journal of Anatomy. 193(1): 105-119. Article link I https://doi.org/10.1046/j.14697580.1998.19310105.x

DiVincenti L, Westcott R, and Lee C (2014). Sheep (Ovis aries) as a model for cardiovascular surgery and management before, during, and after cardiopulmonary bypass. Journal of the American Association for Laboratory Animal Science, 53(5): 439-448. Article link I PMID: 25255065

Emam MA and Abugherin B. (2019). Histological study on the heart ventricle of Egyptian bovines (Bos aegyptiacus). Open veterinary journal. 9(4): 281-286. Article link I DOI: https://dx.doi.org/10.4314\%2Fovj.v9i4.1

Endo H, Tadasu KY, Suzuki N, Suwa G, Uetsuka K, Hashimoto O, Kurohmari M, Hayashi Y (1995). Ultrastructure of Cardiac Myocyte in the Asian Elephant (Elephas maximus). Journal of Veterinary Medical Science, 57(6): 1035-1039. DOI: https://doi.org/10.1292/jvms.57.1035

Frandson RD, Wilke WL, and Fails AD (2009). Anatomy and physiology of farm animals. 7th edition. Wiley-Blackwell, UK. P. 520. Book link 
Gushchin YA and Muzhikyan AA (2014). Influence of fixing fluids on the microscopic structure of organs of small laboratory animals. International Bulletin of Veterinary Medicine, 3: 88-94. Article link

Hooper SL, and Thuma JB (2005). Invertebrate muscles: muscle specific genes and proteins. Physiological Reviews, 85(3):1001-1060. DOI: https://doi.org/10.1152/physrev.00019.2004

Huxley HE, and Haselgrove JC (1977). The Structural Basis of Contraction in Muscle and Its Study by Rapid X-Ray Diffraction Methods. In: Riecker G. et al. (eds) Myocardial Failure. International Boehringer Mannheim Symposia, Springer, Berlin, Heidelberg. DOI: https://doi.org/10.1007/978-3-642-46352-5_2

König HE and Libich HG (2004). Veterinary anatomy of domestic mammals. Textbook and color atlas. Germany: by Schattauer GmbH. P.681. Google Scholar

Kozlovskaya AA. (2014). Morphofunctional features of the structure and development of the valves of the aorta and the pulmonary trunk in ontogenesis. Bulletin of Problems of Biology and Medicine. 1(106): 251-255. link: https://vpbm.com.ua/en/vpbm-2014-01/5726

Kuga SA (2012). Anatomical characteristics of the lungs and hearts in different representatives of the canine family. Hippology and Veterinary Medicine, 2(4): 68-69. Article link

Maximilian Buja L (2007). Anatomy of the Heart. In: Willerson J.T., Wellens H.J.J., Cohn J.N., Holmes D.R. (eds) Cardiovascular Medicine. Springer, London. DOI: https://doi.org/10.1007/978-1-84628-715-2_1

Muzhikyan AA, Makarova MN, Gushchin YA (2014). Features of the pathological study of a group of experimental animals. International Bulletin of Veterinary Medicine, 1: 75-79. Article link

Semchenko VV, Samuseva MV, Moiseeva MV, Kolosov VL (1999). International histological nomenclature (in Latin, Russian and English). Omsk Medical Academy, Russian Federation, p.156.

Vansyatskaya VK and Kirpaneva EA (2014). Morphometric and anatomical features of heart structure of the cattle, pigs and camel. Agriculture - Problems and Prospects, 25: 29-36. Google Scholar

Zelenevsky NV. (2013). International veterinary anatomical nomenclature in Latin and Russian. Fifth edition, St. Petersburg. p. 400. Google Scholar 